\title{
Preliminary study on chronic granulomatous disease in Sri Lanka
}

Shalinda Jude Arjuna Fernando ${ }^{* *} \mathbb{0}$, Noorul Mifra Faiz ${ }^{1}$, Shiroma Mangaika Handunnetti ${ }^{1}$, Aruna Dharshan De Silva², Wasala Mudiyanselage Dhanushka Kumari Dasanayake³, Geethani Devika Wickramasinghe ${ }^{3}$, Rathnayake Mudiyanselage Chandima Hasanthi Karunatilake ${ }^{3}$ and Nilhan Rajiva de Silva ${ }^{3}$

\begin{abstract}
Background: Chronic granulomatous disease (CGD) is a rare primary immunodeficiency of the phagocytic cells, which results in absent or diminished levels of microbicidal reactive oxygen species. The disease occurs due to germline mutations in the genes encoding the five subunits of NADPH oxidase complex. The present study is a pilot study to understand the clinical and genetic aspects of CGD in Sri Lanka.

Methods: Clinical records of thirteen CGD patients were analysed and compared with similar studies performed in different countries and regions to identify patterns in demographics, clinical manifestations and infectious agents. Genomic DNA and CDNA were analysed in eight patients to identify mutations in CYBB and NCF1 genes, thereby to ascertain the potential X-linked and autosomal recessive (AR) CGD patients.

Results: The onset of symptoms in the patient cohort was very early (mean 4.6 months) compared to 20 months in India and 23.9 months in Latin America. Similarly, the age at diagnosis was lower (mean 1.6 years after birth) compared to other studies; 4.5 years in India and 6.1 years in Europe. Pulmonary manifestations were the most common (85\%), followed by skin/subcutaneous infections (77\%) and lymphadenopathy (62\%). The death rate of local patients (38\%) was higher than other countries (India 35\%, Europe 20\%). Majority (77\%) were treated for tuberculosis at some point in life. Genetic analysis confirmed six out of eight patients as X-linked CGD cases with mutations in CYBB gene. A novel splice site mutation was identified in P-07 at position c.141+6 which resulted in the deletion of entire exon 2. Two siblings (P-05 and P-06) from consanguineous parents, were identified with AR-CGD based on the homozygous GT deletion mutation in NCF1 gene.
\end{abstract}

Conclusions: The clinical presentation, manifestations and genetic subtypes in the local cohort, appear to be comparable with global trends. Mycobacterial infections should be investigated and treated with more prominence. Effective treatment options are required to control the high mortality rate.

Keywords: Chronic granulomatous disease, NADPH oxidase, CYBB, NCF1, Mycobacterial infections, X-linked, Autosomal recessive, Sri Lanka

\footnotetext{
*Correspondence: shalinda.fernando90@gmail.com

${ }^{1}$ Institute of Biochemistry, Molecular Biology \& Biotechnology (IBMBB),

University of Colombo, Colombo 03, Sri Lanka

Full list of author information is available at the end of the article
} 


\section{Background}

Chronic granulomatous disease (CGD) is a rare, inherited primary immunodeficiency which affects approximately $1 / 250,000$ worldwide [1]. CGD is categorized into two main subtypes, viz. X-linked and autosomal recessive (AR), based on the mode of inheritance. Overall, about $65 \%$ of CGD cases worldwide are X-linked type while the remaining $35 \%$ are autosomal recessive CGD cases [2]. However, in certain regions with high degrees of consanguineous marriages, AR-CGD is more prevalent [3].

Chronic granulomatous disease is caused by a defect in phagocytic cells (neutrophils, macrophages, and monocytes) which fail to exhibit the 'respiratory burst', the rapid increase in oxidative metabolism following phagocytosis, thereby failing to effectively destroy invading pathogens. This is due to a germ line mutation in one of the five genes which code for the five subunits of the NADPH oxidase enzyme complex [1].

$\mathrm{X}$-linked recessive CGD occurs due to mutations in the $C Y B B$ gene which encodes gp91phox subunit. Mutations in the genes encoding the subunits p47phox, p67phox, p40phox, and p22phox (NCF1, NCF2, NCF4, and CYBA respectively) are responsible for AR-CGD [4]. The most common form of AR-CGD accounting approximately $90 \%$ of all AR-CGD cases is due to mutations in NCF1 gene. The predominant mutation found in these patients is a GT deletion in the GTGT repeat sequence at the beginning of exon 2 of NCF1 [5].

The first CGD case was reported in Sri Lanka in 1999 [6]. There is a paucity of clinical data of CGD patients in Asian countries, especially South Asia. Large multi-centre studies have been carried out in Western countries, and CGD databases have been established in Europe, USA and Latin America. However, Western data might not be applicable for Asian countries due to differences in social practices (e.g. consanguineous marriages) and endemic infections. We report on the patients with CGD in Sri Lanka, and evaluate the clinical scenario and evolution of the disease in a developing country.

\section{Methods \\ Patients}

Ethics clearance was obtained from the Ethics Review Committee of the Medical Research Institute (No $05 / 2016$ and $06 / 2016$ ), and informed written consent was obtained from the parents of the patients.

Thirteen patients with CGD from 11 families were seen at Immunodeficiency Clinic at Medical Research Institute (MRI), Colombo Sri Lanka, from 2004 to December 2017. Their diagnoses were based on $<5 \%$ normal neutrophil activity with the nitroblue tetrazolium (NBT) reduction slide assay. Eleven out of thirteen patients were referred to the Immunodeficiency clinic owing to multiple recurrent infections. The two remaining patients were referred to the clinic, within days of birth due to recurrent infant deaths in the family. Clinical details of the patients, including details of deceased siblings, were obtained from the case records.

\section{Genomic DNA analysis}

Three millilitres of blood was collected into tubes containing EDTA from eight CGD patients after informed written consent was obtained. Genomic DNA was extracted from whole blood stored in EDTA using commercially available QIAmp DNA Blood Mini Kit (QIAGEN, Hilden, Germany) following manufacturer's protocol. Previously described [7] exon specific oligonucleotide primers for NCF1 (2LB2 and 2RB2) were purchased from Integrated DNA Technologies (Iowa, USA). Thirteen sets of exon specific primers for the $C Y B B$ gene were kindly donated by Prof Yu-Lung Lau of University of Hong Kong. The amplified DNA fragments were subjected to bi-directional sequencing with BigDye ${ }^{\circledR}$ Terminator v3.1 cycle sequencing kit and resolved with direct sequencing on an automated fluorescence sequencer, Applied Biosystems ${ }^{\circledR} 3500$ Dx Genetic Sequencer.

\section{CDNA analysis by RT-PCR}

Human total leukocytes were isolated from EDTA-whole blood using dextran sedimentation method, which is based on differential centrifugation principle, as previously described [8]. Total RNA was extracted from separated leukocytes using $\mathrm{TRIzol}^{\circledR}$ reagent as per the manufacturer's guidelines. First-strand cDNA synthesis was performed from total RNA using random primers and the cDNA was immediately amplified into three overlapping fragments. The amplified products were subjected to bi-directional sequencing as described above.

\section{Results}

\section{Demographics and family history}

Eleven patients of the 13 analysed were males while the remaining two were females. The patients originated from 5 districts across the country. The patients comprised of all major ethnic groups of the country, 9 Sinhalese, 2 Tamil, and 2 Muslim patients. The ages of the patients ranged from 3 days to 14 years at the time of sampling. The participants of the present study were diagnosed between 2004 and 2017.

The thirteen CGD patients belonged to eleven families. P-01 and P-12 were brothers while P-05 and P-06 were brother and sister. Four families had consanguineous parents. Four patient families had reported 7 previous sibling deaths (Table 1). All of the sibling deaths occurred during infancy or very young age. In the family of P-01 three elder died due to sepsis. Although not diagnosed, 


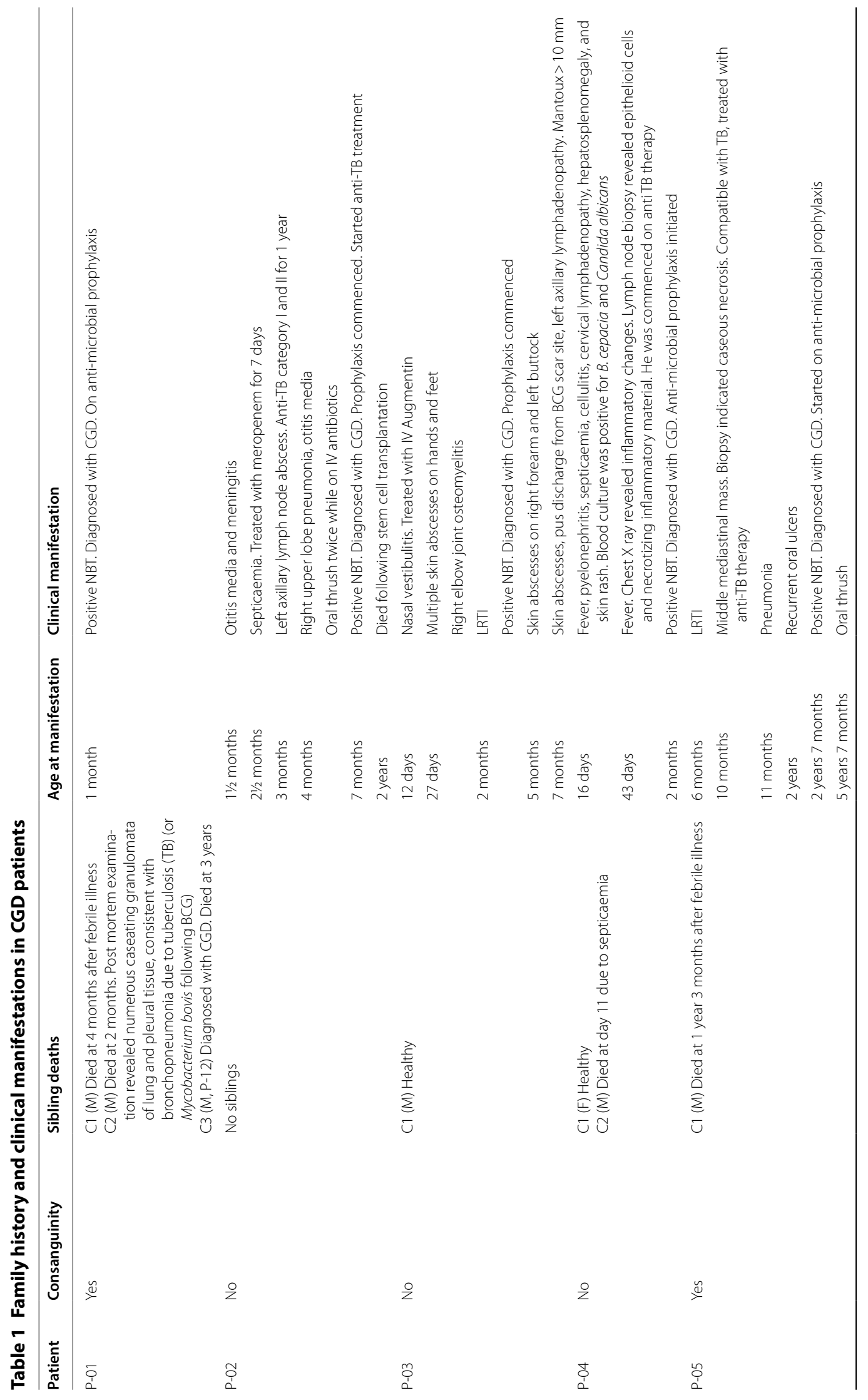




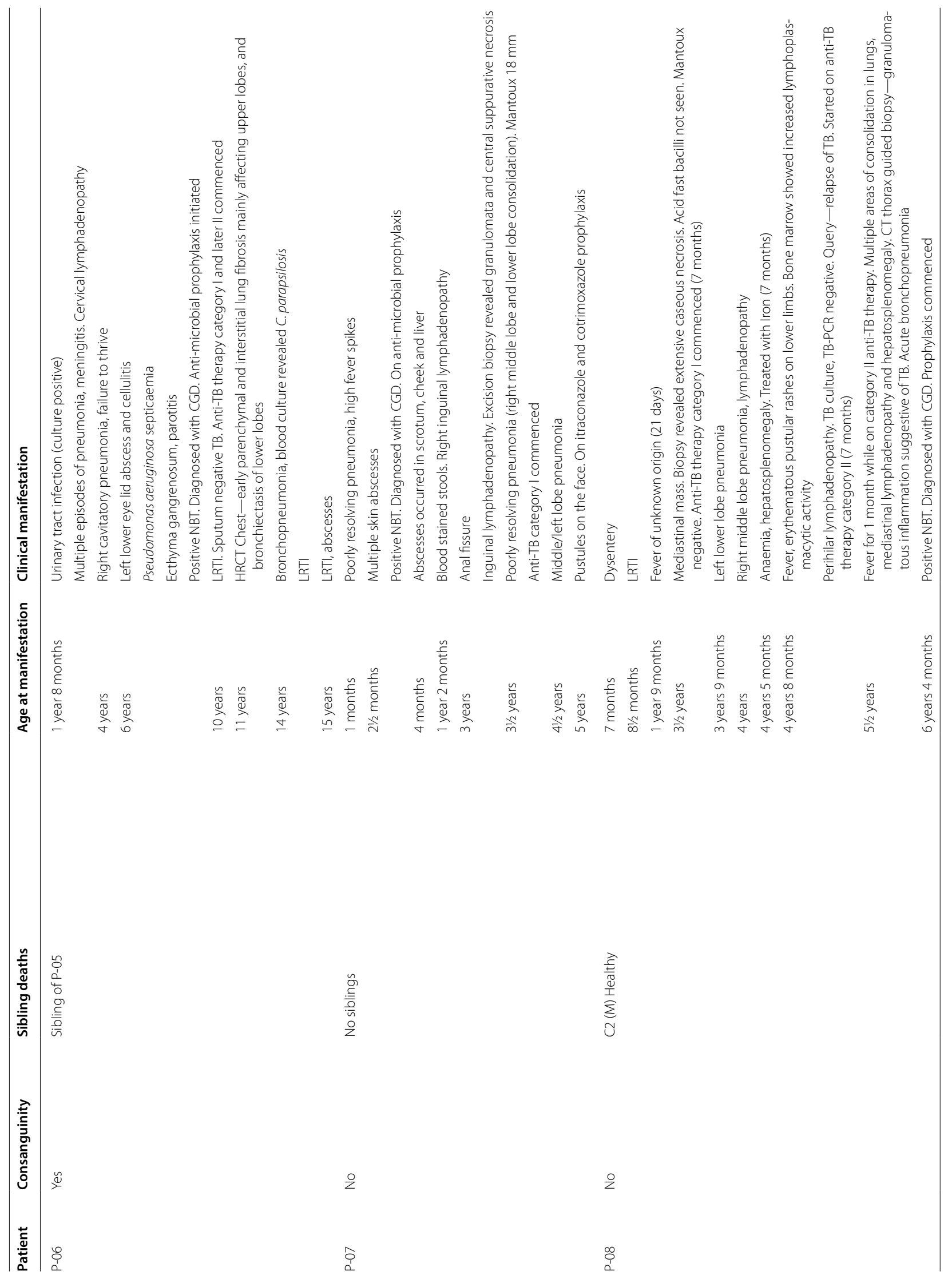




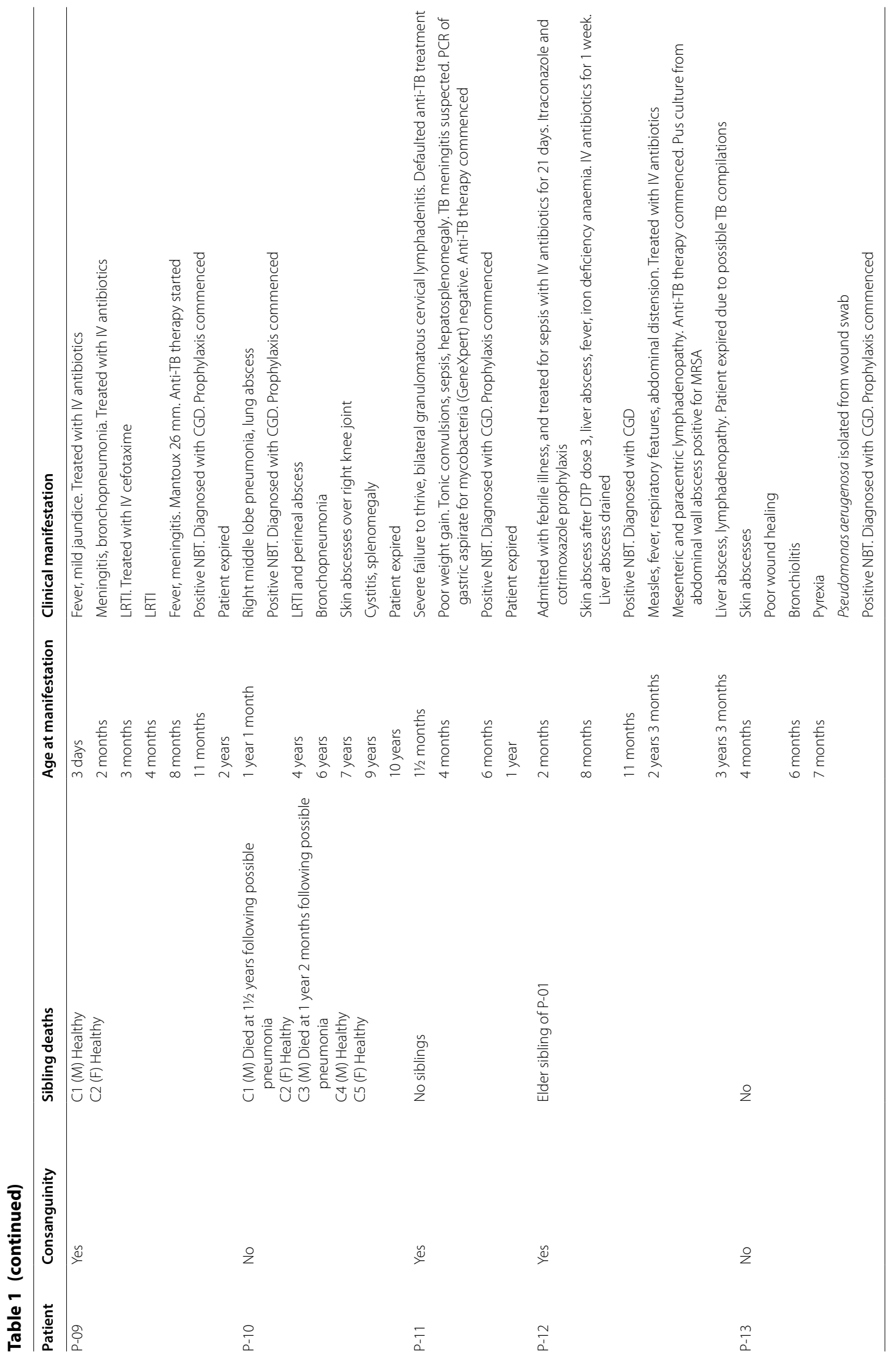


two of the three siblings may have died of complications of CGD. The third sibling had been diagnosed with CGD (P-12), and was on prophylactic treatment. Furthermore, the mother of P-01 (and P-12) reported that three of her siblings passed away during infancy.

\section{Clinical course}

Eleven patients were referred to MRI due to recurrent unresolving infections while P-01 and P-12 (siblings) were referred owing to their family history. The mean age at onset of disease among the patients is $4.6 \pm 5.6$ months. Majority of the patients $(58 \%)$ manifested symptoms as early as 2 months since birth, out of which 3 patients developed symptoms during the first 2 weeks. In X-linked CGD patients, the mean age at onset was $1.5 \pm 1.3$ months and in AR-CGD patients $12 \pm 8.5$ months.

The mean age at diagnosis for the entire cohort was $18.6 \pm 25.8$ months. Most patients (69\%) were diagnosed in infancy. The mean age at diagnosis was $4.6 \pm 3.7$ months in X-linked CGD patients and $51.5 \pm 29.0$ months in AR-CGD patients. The mean diagnostic delay irrespective of subtype was $15.5 \pm 22.8$ months.

Five out of 13 patients (38\%) have died, at an average of $3.65 \pm 3.63$ years. Only 2 of the deceased patients (P-02, $\mathrm{P}-12$ ) are known to have X-linked CGD. P-02 died following stem cell transplantation.

\section{Clinical manifestations}

Respiratory tract was the most prevalent site of clinical manifestations. Eleven patients (85\%) had respiratory tract infections, including bronchopneumonia (69\%). Six patients (46\%) had more than one episode of pneumonia. One patient had bronchiectasis (8\%). After respiratory tract manifestations, the skin was the organ most affected. Ten patients (77\%) developed multiple skin abscesses on the face, neck, and limbs and to some lesser extent skin rashes, ecthyma gangrenosum, and pustules. Other deep seeded abscesses include liver abscesses in 2 patients (15\%) one of whom had 2 episodes, lymph node abscesses in 2 patients (15\%) and lung abscess in one patient (8\%).

Lymph node manifestations were observed in 8 patients (62\%), of which 6 patients had multiple episodes of lymphadenopathy. Meningitis affected 4 patients (31\%), of which one patient developed multiple episodes. Septicaemia was reported in 5 (38\%) patients although the causative pathogen was not identified in the majority.

BCG vaccine was administered at birth to 11 patients (85\%) in the group except for P-01 and P-12 (siblings) owing to their family history of infant deaths. Two patients developed adverse reactions to the vaccine. For instance, P-03 developed an adverse reaction at the BCG scar site along with left axillary lymphadenopathy and a positive Mantoux test, suggestive of possible infection with $M$. bovis. Furthermore, mediastinal masses with caseous necrosis was seen in two patients (P-05 and P-08) indicating disseminated mycobacterial infections. Two patients were diagnosed with tuberculous meningitis, one of whom had a positive Mantoux test $(26 \mathrm{~mm})$. Ten (77\%) patients received anti-TB therapy despite not being able to isolate the organism.

The patients were commenced on prophylaxis antimicrobials; mainly itraconazole and cotrimoxazole, without delay upon their diagnosis. However, 5 (38\%) patients developed multiple infections and manifestations such as LRTI, lymphadenopathy, skin abscesses despite prophylactic treatment. Interferon gamma therapy is not offered in Sri Lanka. One patient (P-02) who underwent stem cells transplantation died following the procedure.

\section{Mutation analysis}

Eight CGD patients (7 males and 1 female) who were laboratory confirmed by slide NBT assay were included in the mutation analysis. Point mutations in $C Y B B$ gene were identified in 06 patients confirming their X-linked CGD status. The detected mutations in $C Y B B$ gene includes, deletions, nonsense and a novel splice site mutation as summarized in Table 2. In P-07 no exonic mutations were identified. However, a novel $\mathrm{T}>\mathrm{A}$ nucleotide substitution (c.141+6T $>$ A) was detected 6 bases downstream from end of exon 2 in the intron 2 region. Analysis of the patient's cDNA revealed that this novel mutation is a pathogenic splice site mutation, which resulted in exon skipping by the deletion of the entire exon 2 (Fig. 1).

The two remaining patients (P-05 and P-06) were classified as AR-CGD cases, based on the detection of the prominent homozygous GT deletion (c.75_76delGT) mutation in NCF1 gene.

\section{Discussion}

The majority of the Sri Lankan patients studied, were X-linked CGD cases (75\%) similar to other studies in Western countries $[1,10,11]$ and in contrast to South Asian (Indian) and other Asian countries such as Turkey and Iran, where AR-CGD is reported with a higher frequency than X-linked CGD [3, 12] (Table 3). Although consanguineous marriages are responsible for this high rates of AR-CGD in Turkey and Iran, consanguinity was not frequent among the Indian patients (2/17), and the higher prevalence of AR-CGD may be due to high incidence of autosomal recessive mutations resulting from marriages being restricted to close-knit communities [2]. Interestingly, 6 patients from 4 families reported to 
have consanguineous parents in the Sri Lankan cohort, although only 2 patients were positively confirmed with AR-CGD.

The overall mortality $(38 \%)$ was higher than in India $(35 \%)$, and in the West $[1,2]$. The mortality in a large cohort of 429 patients from Europe was 20\% [1], whereas in an Italian cohort the mortality was 13\% [13].

The onset of symptoms in the patient cohort was very early (mean $5 \frac{1 / 2}{2}$ months) compared with other studies; 20 months in India [2] and 23.9 months in Latin America [10]. Majority of the CGD patients (85\%) developed at least one unusual or severe infection within the 1st year of life. Seventy seven percent of the local cohort was identified by unusual susceptibility to severe infections before the age of 2 years. In China $90 \%$ of the patients had an onset within 3 months of birth [14]. The mean age at diagnosis of Sri Lankan patients was 1.6 years. This is lower than other countries; India $4 \frac{1}{2}$ years [2], Iran 6.3 years [12], Turkey 4.2 years [3], China 2.24 years [14], Europe 4.9 years (X-linked), 8.8 years (AR) [1]. This may be due to early exposure to infections, genetic phenotype, or easy access to basic healthcare in the country. Additionally, the mean diagnostic delay in local patients was about 15 months, which is significantly lower than the 3.9 year delay in Iran [12].

Large scale CGD studies have shown that the disease manifests earlier in X-linked CGD compared to AR-CGD [1]. Similarly, the mean age of diagnosis is lower in the patients with X-linked CGD compared with those with AR-CGD. For instance, the mean age at diagnosis in patients with X-linked CGD versus AR-CGD are 4.9 vs 8.8 years in Europe, 3.0 vs 7.8 years in USA, and 2.7 vs 5.2 years in Turkey $[1,3,11]$. The mean age at diagnosis in our study was 4.6 months for X-linked CGD while it was 4 years and 3 months for AR-CGD. Despite the small size of the cohort in Sri Lanka, the onset of disease was early (1.5 months) in X-linked CGD patients compared to AR-CGD patients (12 months). All of these findings suggest a milder phenotype or a delay in clinical presentation in AR-CGD.

The clinical presentation and manifestations in the local cohort appear to be comparable with regional and global trends. Most large cohort studies report pulmonary manifestations to be the most common (Table 4). In the local cohort $85 \%$ had lower respiratory tract infections. Seventy seven percent of the patients had skin/

Table 2 Summary of clinical details and genetic analysis of CGD patients

\begin{tabular}{|c|c|c|c|c|c|c|c|c|}
\hline Patient & CGD subtype & $\begin{array}{l}\text { Slide NBT } \\
\text { result (\%) }\end{array}$ & Age at onset & Age at diagnosis & Gene affected & Exon/intron & $\begin{array}{l}\text { Nucleotide } \\
\text { change }\end{array}$ & $\begin{array}{l}\text { Amino acid change } \\
\text { [9] }\end{array}$ \\
\hline P-01 & X-linked & $<5$ & Referred at birth & 1 month & $C Y B B$ & Exon 10 & $\begin{array}{l}\text { Deletion } \\
\text { c.1314delG }\end{array}$ & p.lle439SerfsX63 \\
\hline P-02 & X-linked & $<5$ & 1.5 months & 6.5 months & $C Y B B$ & Exon 11 & $\begin{array}{l}\text { Deletion } \\
\text { c.1415delG }\end{array}$ & p.Gly472AlafsX30 \\
\hline P-03 & X-linked & $<5$ & 1 month & 7 months & $C Y B B$ & Exon 10 & $\begin{array}{l}\text { Deletion } \\
\text { c.1314delG }\end{array}$ & p.lle439SerfsX63 \\
\hline P-04 & X-linked & $<5$ & 16 days & 2 months & $C Y B B$ & Exon 3 & $\begin{array}{l}\text { Nonsense } \\
\text { c.217C > T }\end{array}$ & p.Arg73X \\
\hline P-05 & $A R$ & $<5$ & 6 months & 2.5 years & NCF1 & Exon 2 & $\begin{array}{l}\text { Deletion } \\
\text { c.75_76delGT }\end{array}$ & p.Tyr26Hisfs \\
\hline P-06 & $A R$ & $<5$ & 1.6 years & 10 years & NCF1 & Exon 2 & $\begin{array}{l}\text { Deletion } \\
\text { c.75_76delGT }\end{array}$ & p.Tyr26Hisfs \\
\hline P-07 & X-linked & $1-2$ & 1 month & 2.5 months & $C Y B B$ & Intron 2 & $\begin{array}{l}\text { Splice site } \\
\qquad .141+6 \mathrm{~T}>\mathrm{A}\end{array}$ & $\begin{array}{l}\text { del exon } 2 \\
\text { p.Leu16_Gly47del }\end{array}$ \\
\hline$P-13$ & X-linked & $<5$ & 4 months & 7 months & $C Y B B$ & Exon 3 & $\begin{array}{l}\text { Splice site } \\
\text { c.252G }>\text { A }\end{array}$ & $\begin{array}{l}\text { del. exon } 3 \\
\text { p.Ser48_Ala84del }\end{array}$ \\
\hline
\end{tabular}

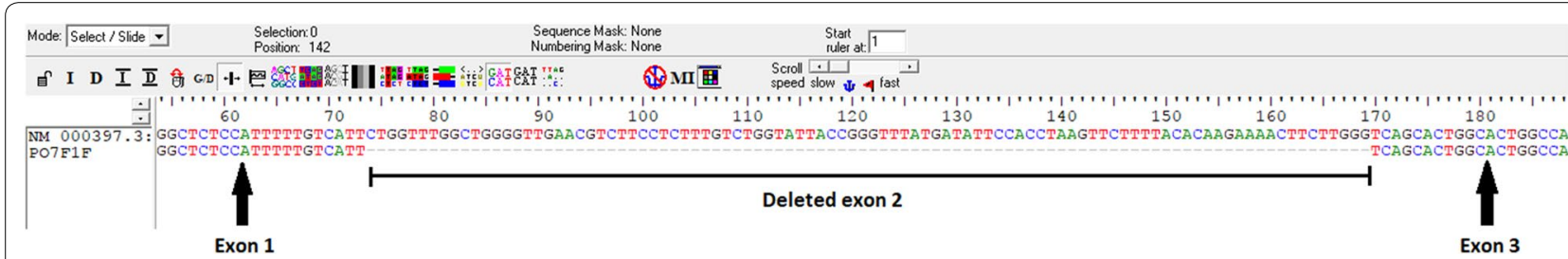

Fig. 1 Exon skipping in P-07. The novel splice site mutation identified resulted in the deletion of the entire exon 2 region of the CYBB CDNA in P-07. The figure shows the deleted region when the patient CDNA (bottom) is aligned with a reference sequence (top) (NCBI Accession: NC_000023.9) 
Table 3 Comparison of patient cohort demographics of different CGD studies

\begin{tabular}{|c|c|c|c|c|c|c|c|}
\hline \multirow[t]{2}{*}{ Country/region } & \multirow{2}{*}{$\begin{array}{l}\text { Number } \\
\text { of patients }\end{array}$} & \multicolumn{2}{|c|}{ Gender } & \multirow[t]{2}{*}{ Consangui-nity } & \multicolumn{2}{|c|}{ CGD subtype } & \multirow[t]{2}{*}{ Deaths } \\
\hline & & Male & Female & & X-linked & AR & \\
\hline Sri Lanka & 12 & 10 & 2 & $6(46 \%)$ & $6(75 \%)$ & $2(25 \%)$ & $4(38.4 \%)$ \\
\hline India [2] & 17 & 15 & 2 & $2(11.7 \%)$ & 7 (41\%) & $10(59 \%)$ & $6(35 \%)$ \\
\hline Iran [12] & 41 & 29 & 12 & $23(56.1 \%)$ & & & $5(12.2 \%)$ \\
\hline Turkey [3] & 89 & 64 & 25 & $42(57.5 \%)$ & $34(38.2 \%)$ & $55(61.8 \%)$ & $9(10.1 \%)$ \\
\hline China [14] & 48 & 44 & 4 & 0 & $36(75 \%)$ & $3(6 \%)$ & $11(22 \%)$ \\
\hline Latin America [10] & 71 & 58 & 13 & - & $53(74.6 \%)$ & $18(25.3 \%)$ & - \\
\hline Europe [1] & 429 & 351 & 78 & - & $290(67 \%)$ & 139 (33\%) & $84(20 \%)$ \\
\hline USA [11] & 368 & 316 & 52 & - & 259 (70\%) & 81 (22\%) & 65 (17.6\%) \\
\hline
\end{tabular}

subcutaneous infections while $62 \%$ reported lymph node involvement. These figures seem to correlate with other worldwide figures $[1,3,10]$. Gastrointestinal manifestations such as inflammatory bowel disease were not seen in our patients. In addition, chronic lung disease, including bronchiectasis and fibrosis was seen in only one patient. This is in contrast to other cohorts, where it is more common [15].

The spectrum of microorganisms implicated in causing infections may vary slightly in different regions, but the most common pathogens reported worldwide are Staphylococcus aureus, Burkholderia cepacia, Serratia marcescens, Nocardia, and Aspergillus sp. [16]. The Indian study reports Aspergillus to be the most common organism isolated in patients followed by Staphylococcus aureus, Burkholderia cepacia and Candida [2]. However, in Sri Lanka, the causative agent had not been isolated in majority of instances. Due to lack of facilities, bronchoscopy or direct lung biopsies are not routinely done. The patients were often managed in peripheral hospitals, with no input from an Immunologist. Many of the infectious complications occurred before an Immunological diagnosis. B. cepacia and Candida were isolated from blood in P-04. Candida was also isolated from an oral swab two patients with oral thrush, while, septicaemia caused by Pseudomonas was reported in P-06. Pseudomonas aerugenosa was isolated from a wound swab from P-13. Aspergillus sp. was the main pathogen responsible for pulmonary infections in CGD patients in Europe, France, Turkey, and India [1-3, 17]. The lack of reported Aspergillus infections in the Sri Lankan cohort is surprising and highly unlikely, suggesting more thorough microbiological examinations are required.

The clinical data collected in the Asian Primary Immunodeficiency (APID) network database suggest that Mycobacteria tuberculosis and M. bovis infections are significant in Southeast Asian CGD cohorts [18]. Furthermore, a retrospective study on CGD patients with mycobacterial disease from Latin America, Africa, Europe, and Asia, revealed that $44 \%$ had TB [19]. In the Chinese cohort of 17 patients, 7 had TB while 8 had BCG complications [20]. Pulmonary tuberculosis was recorded as the second common respiratory involvement (31.7\%) in the Iranian cohort [12]. In the Indian cohort, Mycobacterium was detected in only one patient, while two more were treated for TB [2].

In Sri Lanka, ten (77\%) CGD patients received antiTB medication at some point in their treatment. The decisions to treat were based on biopsy findings in 5 patients, Mantoux positivity in 3 patients, and X-ray evidence in 2 patients. Facilities for AFB staining, Mantoux, culture, PCR and T spot (IGRA) are available in Sri Lanka, but are not routinely done. Using the acid fast stain on biopsies is generally not done, unless specially requested. Culture is generally done with sputum, but not generally from tissues. In addition, differentiation of $M$. bovis from $M$ tuberculosis is not done. PCR (GeneXpert) is performed, but not done routinely due to the cost. On a few occasions PCR and culture were performed. Most often, it was a clinical diagnosis based on BCG immunization, location of lymphadenopathy, presence of necrosis/caseation, and Mantoux $(>10 \mathrm{~mm})$. Interestingly, none of the patients were positive for Mycobacterium cultures. TB PCR and acid fast staining were negative. BCG vaccine is given to all infants at birth, and the coverage is $99 \%$ in the country [21]. In our cohort, BCG vaccine was given to $11 / 13$ patients. While facilities are available for categorizing BCG infection as definitive, probable, possible, and ruled out (European Society for ImmunodeficienciesESID criteria) [22], it was not availed of in all instances. In many instances, the diagnosis of BCG/mycobacterial infection was done prior to referral to our clinic. With the clinical history, it is probable that the positive Mantoux was due to infection with M. bovis. The diagnosis would fit the possible category (ESID criteria). The 
Table 4 Comparison of clinical manifestations in CGD patients

\begin{tabular}{|c|c|c|c|c|c|c|c|c|}
\hline \multirow[t]{2}{*}{ Site of clinical manifestation } & \multicolumn{8}{|c|}{ Prevalence in different countries/regions-proportion (percentage) (\%) } \\
\hline & Sri Lanka & India [2] & Iran [12] & Turkey [3] & China [14] & $\begin{array}{l}\text { Latin } \\
\text { America } \\
{[10]}\end{array}$ & Europe [1] & USA [11] \\
\hline Lung & 85 & 82 & 66 & 83 & 77 & 77 & 66 & 79 \\
\hline Pneumonia & 81 & & & & & & & \\
\hline Pulmonary TB & 18 & & & & & & & \\
\hline Lung abscess & 9 & & & & & & & \\
\hline Lung fibrosis/bronchiectasis & 27 & & & & & & & \\
\hline Skin/subcutis & 77 & 47 & 63 & 45 & 46 & 42 & 53 & 42 \\
\hline Lymph nodes & 62 & 82 & 76 & 65 & 50 & 59 & 50 & 53 \\
\hline Liver & 46 & 59 & - & 63 & 8 & - & 32 & 27 \\
\hline Liver abscess & 33 & & & & & & & \\
\hline Hepatosplenomegaly & 83 & & & & & & & \\
\hline Gl tract & 31 & 35 & 56 & 22 & 54 & 42 & 48 & - \\
\hline Diarrhoea/dysentery & 50 & & & & & & & \\
\hline Oral ulcers & 25 & & & & & & & \\
\hline Parotitis & 25 & & & & & & & \\
\hline Urinary tract & 23 & 6 & - & 22 & 2 & 20 & 22 & 10 \\
\hline Septicaemia & 38 & 25 & - & 30 & 23 & 23 & 20 & 18 \\
\hline Ear & 8 & 12 & 24 & 22 & 8 & 29 & 14 & - \\
\hline Osteomyelitis & 8 & 18 & 29 & 23 & 4 & 16 & 13 & 25 \\
\hline Meningitis & 31 & - & 2 & 17 & 4 & - & 7 & 4 \\
\hline TB meningitis & 25 & & & & & & & \\
\hline BCG complications & 8 & - & 17 & 22 & 53 & 30 & 8 & - \\
\hline
\end{tabular}

The most common manifestations reported in each cohort are indicated in italic text

BCG strain used the vaccine in Sri Lanka is sensitive to all drugs except pyrazinamide. As the 4 drugs are given in one capsule, pyrazinamide is also administered. The duration for anti TB therapy was the standard regime. ESID suggests 3 drugs for BCG it is in patients with SCID, and continuation till complete immunological reconstitution occurs after HSCT (in SCID for example). For BCGosis, anti-TB treatment including four or more anti-TB drugs should be given until the patient fully recovers, followed by two drugs complete immunological reconstitution after HSCT is achieved. While there are no guidelines for CGD, prolonged therapy was not given. This may have been responsible for the increased mortality.

Genetic analysis of eight Sri Lankan CGD patients revealed that two siblings (P-05 and P-06) had p47phox AR-CGD resulting from the prominent GT deletion in exon 2 of NCF1 gene. The remaining six patients were classified as X-linked CGD. Five patients had previously described [9] CYBB mutations. The splice site mutation $(\mathrm{c} .141+6 \mathrm{~T}>\mathrm{A})$ identified in the remaining patient (P-07) was a novel mutation. Splice site mutations have been known to be responsible for many genetic diseases including $\beta$-thalassemia and CGD [23]. Mutations located in both donor and acceptor splice sites may results in exon skipping and shortened polypeptides. Splice site mutations appear to be a common cause of X-linked CGD with $17.6 \%$ of 681 reported mutations falling into this category [9].

The investigators encountered several limitations during the study which are worth mentioning. The Immunodeficiency Clinic at the MRI in Colombo is the sole clinic of its kind to diagnose CGD and other immunological disorders. Patients are referred from across the country to be investigated and diagnosed. The authors believe the prevalence of CGD in the country could be higher, and that a percentage of patients remain unreported and undiagnosed. Paucity of knowledge about primary immune deficiency among medical staff is one problem. Lack of resources to investigate and accurately diagnose infectious pathogens especially BCG disease and TB has contributed to the high mortality rate.

The CGD diagnosis in this study was based on the qualitative slide NBT assay. This assay is now being replaced by the flow cytometry based Dihydrorodemine (DHR) assay as the slide NBT assay is prone to 
observer bias and requires expertise. The DHR assay has not yet been established and validated in the country due to lack of resources. Furthermore, only eight patients of the cohort were sequenced. Four out of the five remaining patients could not be sequenced as they were already deceased by the time the genetic assay was established and validated, whereas the remaining patient is yet to be sequenced.

\section{Conclusions}

The Sri Lankan CGD patient cohort displayed classic clinical manifestation associated with CGD with pulmonary infections being the most common. Patients suffered recurrent severe infection despite using antimicrobial prophylaxis. Mycobacterial infections are potentially lethal in CGD patients and BCG vaccination should be avoided in infants with the disease. Despite the low number of patients to draw definite conclusions, mortality rate in Sri Lankan cohort remains high. The onset of disease and age at diagnosis is low in comparison to regional countries. Furthermore, the findings support the notion that X-linked CGD manifests earlier and is more severe than AR-CGD. An important highlight of the study was the identification of a novel $C Y B B$ pathogenic mutation.

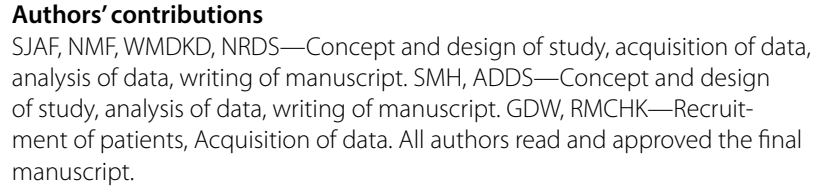
analysis of data, writing of manuscript. SMH, ADDS-Concept and design of study, analysis of data, writing of manuscript. GDW, RMCHK-Recruitment of patients, Acquisition of data. All authors read and approved the final manuscript.

\section{Author details}

${ }^{1}$ Institute of Biochemistry, Molecular Biology \& Biotechnology (IBMBB), University of Colombo, Colombo 03, Sri Lanka. ${ }^{2}$ Genetech Research Institute, Colombo 08, Sri Lanka. ${ }^{3}$ Department of Immunology, Medical Research Institute (MRI), Colombo 08, Sri Lanka.

\section{Acknowledgements}

We acknowledge study participants, staff of Department of Immunology, MRI for patient recruitment and clinical data analysis, staff of IBMBB, University of Colombo for laboratory facilities, and Prof Yu Lung Lau of University of Hong Kong for generously providing CYBB primers.

\section{Competing interests}

The authors declare that they have no competing interests.

\section{Availability of data and materials}

All data generated or analysed during this study are included in this published article and are available from the corresponding author on reasonable request. The reference DNA sequence for CYBB is available at NCBI, https:// www.ncbi.nlm.nih.gov/nuccore/NC_000023.9/.

\section{Consent for publication}

Informed written consent was obtained from the parents of the patients.

\section{Ethics approval and consent to participate}

Ethics clearance was obtained from the Ethics Review Committee of the Medical Research Institute (No 05/2016 and 06/2016).

\section{Funding}

Funding was obtained from Masters' research allocations of IBMBB students SJAF and NMF and supplemented with MRI Financial Grants (05/2016 and 06/2016).

\section{Publisher's Note}

Springer Nature remains neutral with regard to jurisdictional claims in published maps and institutional affiliations.

Received: 27 March 2018 Accepted: 8 May 2018

Published online: 17 September 2018

\section{References}

1. van den Berg J, van Koppen E, Åhlin A, Belohradsky B, Bernatowska E, Corbeel $\mathrm{L}$, et al. Chronic granulomatous disease: the European experience. PLoS ONE. 2009:4(4):e5234. https://doi.org/10.1371/journal.pone.00052 34.

2. Rawat A, Singh S, Suri D, Gupta A, Saikia B, Minz R, et al. Chronic granulomatous disease: two decades of experience from a tertiary care centre in North West India. J Clin Immunol. 2013:34:58-67.

3. Koker M, Camcioglu Y, van Leeuwen K, Kilic S, Barlan I, Yilmaz M, et al. Clinical, functional, and genetic characterization of chronic granulomatous disease in 89 Turkish patients. J Allergy Clin Immunol. 2013. https:// doi.org/10.1016/j.jaci.2013.05.039.

4. Roos D, de Boer M. Molecular diagnosis of chronic granulomatous disease. Clin Exp Immunol. 2013;175:139-49.

5. Hayrapetyan A, Dencher PCD, van Leeuwan K, de Boer M, Roos D. Different unequal crossover events between NCF1 and its pseudogenes in autosomal p47phox deficient chronic granulomatous disease. Biochim Biophys Acta. 2013;1832:1662-72.

6. Detrick B, Hamilton RG, Folds JD. Manual of molecular and clinical laboratory immunology. 7th ed. Washigton DC: American Society for Microbiology; 2006.

7. Berends C, Dijkhuizen B, de Monchy JGR, Gerritsen J, Kauffman HF. Induction of low density and up regulation of CD 11 b expression of neutrophils and eosinophils by dextran sedimentation and centrifugation. J Immunol Methods. 1994;167:183-93.

8. Lucas GN, Sriranganathan S, Gunewardene S, de Silva NR. A case of chronic granulomatous disease. Sri Lankan J Child Health. 2000;29:27-8.

9. Roos D, Kuhns DB, Maddalena A, Roesler J, Lopez JA, Ariga T, et al. Hematologically important mutations: X-linked chronic granulomatous disease (third update). Blood Cell Mol Dis. 2010;45:246-65.

10. de Oliveira-Junior EB, Zurro NB, Prando C, Cabral-Marques O, Pereira PVS, Schimke LF, et al. Clinical and genotypic spectrum of chronic granulomatous disease in 71 Latin American patients: first report from the LASID registry. Pediatr Blood Cancer. 2015;62:2101-7.

11. Winkelstein JA, Marino MC, Johnston RB, Boyle J, Curnutte J, Gallin JI, et al. Chronic granulomatous disease. Report on a national registry of 368 patients. Medicine (Baltimore). 2000;79:155-69.

12. Movahedi M, Aghamohammadi A, Rezaei N, Shahnavaz N, Jandaghi AB, Farhoudi $A$, et al. Chronic granulomatous disease: a clinical survey of 41 patients from the Iranian primary immunodeficiency registry. Int Arch Allergy Immunol. 2004;134:253-9.

13. Di Matteo G, Giordani L, Finocchi A, Ventura A, Chiriaco M, Blancato J, et al. Molecular characterization of a large cohort of patients with chronic granulomatous disease and identification of novel CYBB mutations: an Italian multicenter study. Mol Immunol. 2009:46:1935-41.

14. Wu J, Wang W, Zhang Y, Chen T. Clinical features and genetic analysis of 48 patients with chronic granulomatous disease in a single center study from Shanghai, China (2005-2015): new studies and a literature review. J Immunol Res. 2017. https://doi.org/10.1155/2017/8745254.

15. Wolach B, Gavrieli R, de Boer M, van Leeuwen K, Berger-Achituv S, Stauber T, et al. Chronic granulomatous disease: clinical, functional, molecular, and genetic studies. The Israeli experience with 84 patients. Am J Hematol. 2017;92:28-36.

16. Song E, Jaishankar GB, Saleh H, Jithpratuck W, Sahni R, Krishnaswamy G. Chronic granulomatous disease: a review of the infectious 
and inflammatory complications. Clin Mol Allergy. 2011. https://doi. org/10.1186/1476-7961-9-10.

17. Beauté J, Obenga G, Le Mignot L, Mahlaoui N, Bougnoux ME, Mouy R, et al. Epidemiology and outcome of invasive fungal diseases in patients with chronic granulomatous disease: a multicenter study in France. Pediatr Infect Dis J. 2011;30:57-62.

18. Lee PW, Lau YL. Endemic infections in Southeast Asia provide new insights to the phenotypic spectrum of primary immunodeficiency disorders. Asian Pac J Allergy Immunol. 2013;31:217-26.

19. Conti F, Lugo-Reyes SO, Galicia LB, He J, Aksu G, de Oliveira EB Jr, et al. Mycobacterial disease in patients with chronic granulomatous disease: a retrospective analysis of 71 cases. J Allergy Clin Immunol. 2016. https:// doi.org/10.1016/j.jaci.2015.11.041.

20. Lee PPW, Chan K-WC, Jiang L, Chen T, Chengrong Li, Lee TL, et al. Susceptibility to mycobacterial infections in children with $X$-linked chronic granulomatous disease. A review of 17 patients living in a region endemic for tuberculosis. Pediatr Infect Dis J. 2008;27:224-30.

21. Wijesinghe PR, Palihawadana P, De Alwis S, Samaraweera S. Annual risk of tuberculosis infection in Sri Lanka: a low prevalent country with a high $B C G$ vaccination coverage in the South-East Asia Region. WHO South East Asia J Public Health. 2013;2:34-40.

22. European Society for Immunodeficiencies. Diagnostic criteria for PID. https://esid.org/Working-Parties/Clinical/Resources/Diagnostic-criteriafor-PID2\#Q2. Accessed 30 Apr 2018

23. De Boer M, Bolscher BGJM, Dinauer MC, Orkin SH, Smith CIE, Ahlin A, et al. Splice site mutations are a common cause of X-linked chronic granulomatous disease. Blood. 1992;80:1553-8.
Ready to submit your research? Choose BMC and benefit from:

- fast, convenient online submission

- thorough peer review by experienced researchers in your field

- rapid publication on acceptance

- support for research data, including large and complex data types

- gold Open Access which fosters wider collaboration and increased citations

- maximum visibility for your research: over $100 \mathrm{M}$ website views per year

At BMC, research is always in progress.

Learn more biomedcentral.com/submissions 